\title{
Le possible : alors et maintenant. Comment penser avec et sans Foucault autour du droit pénal et du droit public
}

The Possible, now and then. How to think public law and criminal law with and without Foucault?

\section{Colin Gordon}

\section{(2) OpenEdition Journals}

\section{Édition électronique}

URL : http://journals.openedition.org/conflits/18899

DOI : 10.4000/conflits. 18899

ISSN : $1777-5345$

\section{Éditeur :}

CCLS - Centre d'études sur les conflits lilberté et sécurité, L'Harmattan

\section{Édition imprimée}

Date de publication : 31 décembre 2014

Pagination : 111-134

ISBN : 978-2-343-05760-6

ISSN : $1157-996 X$

\section{Référence électronique}

Colin Gordon, « Le possible : alors et maintenant. Comment penser avec et sans Foucault autour du droit pénal et du droit public », Cultures \& Conflits [En ligne], 94-95-96 | été-automne-hiver 2014, mis en ligne le 20 février 2016, consulté le 30 mars 2021. URL : http://journals.openedition.org/conflits/18899 ; DOI : https://doi.org/10.4000/conflits.18899 


\section{Le possible : alors et maintenant Comment penser avec et sans Foucault autour du droit pénal et du droit public}

\section{Colin GORDON 1}

Colin Gordon est un spécialiste de Michel Foucault et a très largement contribué à la diffusion des travaux de celui-ci dans le monde anglophone, en tant que traducteur, éditeur et commentateur. Il a publié dans Critique, Esprit, Le magazine littéraire et Aut Aut. Il est le coéditeur du recueil The Foucault Effect. Studies in Governmentality, paru aux Presses universitaires de Chicago en 1991.

T histoire du présent, telle qu'elle a été inventée et pratiquée par Michel Loucault, est un défi à ce qui est, et à ce que nous sommes. Elle présente de nous-mêmes une image que nous ne pouvons ni renier, ni assumer. Surveiller et punir a réussi, à propos de l'ordre judiciaire et de la justice criminelle dans nos sociétés, à nous donner à voir justement ce qui se pratiquait, au nom de la punition. L'ouvrage a contribué à questionner l'acceptabilité des pratiques punitives contemporaines.

Relayant une leçon de Nietzsche, Foucault a démontré que nos raisons de punir sont historiquement variables et fugaces ; il a fait en sorte que la punition redevienne pour nous une question, en tous les sens, critique. En ouvrant une brèche dans un régime de légitimité et de rationalité, le texte de Foucault a dégagé des possibilités de questionnement et de changement : changement dans l'ordre des choses et changement de ce que nous sommes.

Mais, qu'est devenu ce possible d'alors ? On peut dire que, d'une certaine manière, le diagnostic généalogique a été " mis à l'épreuve " (en empruntant ici la notion forgée par Foucault plus tard) de la réalité politique. Seulement, quels en sont les résultats ?

1. Mes vifs remerciements à l'équipe de rédaction (Amandine Scherrer, Didier Bigo), ainsi qu'à Fabienne Brion, Graham Burchell, Philippe Chevallier et Yvon Lochard. 
Il y aurait sans doute une histoire à faire de ce possible d'alors, avec tous ses aléas, son kairos, ses occasions. Foucault parle en Mai 1981 d'un sentiment des individus, engagés dans des luttes, mais non dans la politique institutionnelle, qui arrivaient néanmoins à se reconnaître dans la victoire de la gauche. Il considère alors qu'il ne faut pas mépriser ce sentiment qui fait ce qu'il appelle «la victoire d'une possibilité 2 ».

Dans cette histoire du possible, il faudrait, d'une part, analyser ce que fut, et ce que devint ce possible-là : les volontés de changement qu'il mobilisa, les résistances qu'il affronta, ses impacts et ses acquis. D'autre part, il faudrait nous demander ce qu'il en est de notre possible à nous aujourd'hui, et ce que nous pouvons éventuellement apprendre à propos de notre propre présent, en utilisant les méthodes et les outils de Foucault, et en les révisant ou en les adaptant, le cas échéant.

\section{Le possible, maintenant?}

Nous vivons certainement un autre présent, même si, à bien des égards, il y a entre notre actualité et celles de 1975 et 1981 des similitudes encore (sinon trop) reconnaissables. Mais les différences et les nouveautés ne manquent pas - légitimations modifiées, formes prises par les états d'exception, problématisations et dysfonctions différenciées. Nous aurons à les prendre en compte.

Pour aborder ces deux tâches, on propose ici quelques bribes de réflexion, quelques propositions, et quelques conclusions très partielles que je résume ici rapidement par avance.

L'épreuve du réel pour un texte, si tant est qu'il ait pu avoir un quelconque effet dans le réel, consiste bien en partie dans l'épreuve par l'opinion. Dès lors, pour comprendre les enjeux de cette épreuve, il faut regarder plus loin que ce seul livre célèbre, pour prendre en compte notamment l'ensemble des positions de Foucault à l'égard du droit et du juridique en général. Nous pouvons, en outre, étendre nos lectures grâce aux ajouts majeurs tirés des éditions posthumes, aujourd'hui encore inachevées. Mais, notons que malgré l'importance de Surveiller et Punir dans sa vie et son œuvre, il nous faut admettre que le droit, même s'il se retrouve un peu partout dans ses écrits, n'est pas le thème majeur du travail de Foucault, et que Foucault ne s'est jamais donné pour tâche de proposer une pensée unifiée du droit. Il est pourtant pertinent de s'interroger sur les constantes, les continuités qui se trouvent dans sa pensée à ce sujet, et d'examiner les déplacements ou éventuellement les ruptures qui la parcourent.

2. On peut d'ailleurs estimer que, par ses travaux et ses interventions, Foucault a contribué, au moins très modestement, à cette « victoire». 
Cette pensée multiple s'est voulue, au moins parfois, polémique. Là où elle a touché à quelques points névralgiques dans son actualité, elle a aussitôt suscité des contre-lectures, parfois largement acceptées. Dès lors, quelle est la vérité de ces contre-vérités sur le rapport de Foucault au droit ? Et si l'on se tourne vers notre actualité, connaît-on vraiment si bien le contenu pertinent de son travail, ou du travail qu'il a rendu possible, sur ces thèmes, ainsi que le contenu effectif de sa fameuse boîte à outils ? Rien n'est moins sûr.

À partir de ce constat, il est possible d'émettre deux propositions méthodologiques. Premièrement, il est important de retrouver les suites, souvent oubliées, de la continuation du travail de Surveiller et Punir, par Foucault luimême et par ses co-chercheurs (travaux rendus injustement trop méconnus par les effets et les aléas de diaspora du groupe). Deuxièmement, il est nécessaire aussi de retrouver la part qui revient aux enjeux du droit et des domaines de juridiction dans les travaux de Foucault, qui n'ont été pour une large part que très récemment disponibles, et qui relèvent de la période de son « trip gréco-latin » entre 1980 et 1984, ainsi que du contenu éventuel, trop souvent décrit comme impénétrable, de l'agenda qui aurait dû prolonger son travail.

Il est à cet égard frappant de remarquer que l'édition posthume des cours du Collège de France, en rénovant radicalement la connaissance et compréhension de son travail, a d'ailleurs valu à Foucault, sous la plume de plusieurs commentateurs, d'être considéré comme un penseur prémonitoire ou prescient, en ce qui concerne notamment ses analyses sur le néolibéralisme, le biopolitique et/ou le sécuritaire. Mais, est-ce bien le cas et qu'en est-il de cet apport soi-disant «prescient » en ce qui concerne le domaine de la pénalité ? Quels outils de sa boîte méthodologique sont encore utilisables pour les besoins d'une autre actualité ? En sus de réponses brèves à ces questions, je ferai, en conclusion, quelques propositions concernant le possible maintenant.

\section{Foucault et le droit en général}

\section{Constantes et négations}

La question des décalages, théoriques et pratiques, entre le droit et l'ordre, entre la loi et le gouvernement, transparaît clairement dans l'œuvre de Foucault depuis Histoire de la Folie. Entre 1971 et sa mort, en passant par le GIP (Groupe information prison), l'avortement, les débats sur la peine de mort, les discussions avec le Syndicat de la Magistrature, les QHS (Quartiers de haute sécurité), le droit d'asile et le droit de la défense, la ligne de préoccupations avec des enjeux pratiques et théoriques dans ce domaine juridicopénal, en particulier avec la question des droits et des peines applicables, est continue et serrée. L'apport philosophique de Foucault au cours de ces années passe notamment par de petits textes, dits d'intervention ponctuelle, qui ont néanmoins dans sa réflexion une densité et une portée à la fois surprenantes et 
inhabituelles. Son action donnera lieu d'ailleurs à des initiatives et des collaborations dont la persistance peut se tracer, au-delà de sa mort, jusqu'à nos jours.

Cette action a ses constantes, elle a aussi ses déplacements, et elle ne manque jamais d'un élément de tension dans son rapport à l'actualité. Afin d'éclairer ces tensions, il nous faut d'abord rappeler rapidement les divers moments et les manières dont Foucault a envisagé le décalage entre son propre champ d'analyse et l'ordre du juridique, avant de voir les relations entre le modèle juridico-discursif, les formes de gouvernementalité et les modes de véridiction.

Contre le "juridico-discursif » : dans La Volonté de Savoir, Foucault met dos à dos, pour les refuser toutes les deux, l'« hypothèse répressive » sur la sexualité et la conception du pouvoir conçu selon un modèle juridique, à savoir le modèle d'un énoncé impératif qui interdit ce qui sera ensuite réprimé et puni. Notre analyse politique, qui est encore « jeune » et qui n'a pas encore su couper la tête du roi, est dès lors invitée à dépasser ce modèle régalien et légaliste du pouvoir. Cela implique de comprendre :

- que le droit peut être inutile ou illusoire. Foucault dit (au moins de temps en temps) que l'acquisition ou l'élargissement des droits, considérés comme cible de luttes politiques ou de résistances au pouvoir, peuvent être un leurre.

- qu'entre droit et pouvoir, les rapports sont hétérogènes. Foucault indique (notamment dans Surveiller et punir, mais en se corrigeant partiellement ensuite dans Sécurité, Territoire, Population) que l'ordre des libertés formelles dans la société industrielle est une apparence trompeuse, servant à dissimuler la réalité de pouvoirs disciplinaires et normatifs qui, eux, ne relèvent pas d'un ordre juridique; ordre qu'ils ont plutôt tendance à dénaturer et parasiter.

- que la gouvernementalité peut s'opposer à la légitimation. Dans un cours de 1978, Foucault introduit pour définir l'objet de son analyse historique la notion de gouvernementalité, c'est à dire la rationalité de l'action gouvernementale, conçue dans sa différence et sa distinction vis-à-vis du principe et des fondements de la légitimité et de l'institution juridique de la souveraineté : ce qui ne veut pourtant pas dire que la question de la souveraineté légitime n'ait pas son histoire à elle, en interférence constante avec celle de la gouvernementalité.

- qu'il y a des « coups d'État permanents». En reprenant, sans doute avec un grain de malice, le titre d'un livre de François Mitterrand, Foucault décrit comme « coup d'État permanent » le mode d'action caractéristique, au début 
de la modernité, du premier avatar de la gouvernementalité qu'est la raison d'État, agissant dans le cadre de l'État de police et du cadre économique des politiques mercantilistes. Ce gouvernement agit, selon Foucault, plutôt par des règlements que par des lois; il agit impérieusement, directement, ponctuellement, imprévisiblement, selon les nécessités tactiques des cas et des circonstances.

Le débat entre Michel Foucault et Noam Chomsky, dans une émission de télévision hollandaise en novembre 1971, débat qui vient d'être réédité par son modérateur Fons Elders, avec l'ajout en ligne d'un entretien inédit, nous permet d'y ajouter d'autres éléments ${ }^{3}$. Comme ce texte n'est pas paru en France du vivant de Foucault (il n'est même pas certain qu'il ait approuvé sa parution en anglais) et que ce dernier y prend quelques positions qu'il ne paraît pas avoir ensuite maintenues, il faut être prudent. Mais le cœur de ce débat est intéressant car il tient au désaccord entre Foucault et Chomsky sur le fait que, pour Foucault, les enjeux des luttes contre l'oppression et la domination ne sont pas utilement exprimés à travers les revendications des droits de l'homme, des droits humains. Les raisons de ce refus de la part de Foucault sont tout autant antijuridiques qu'antihumanistes. Foucault objecte ici que le langage des droits fait partie d'un système d'ordre bourgeois, où la justice se trouve faussement présentée comme une instance neutre et impartiale d'instruction et de règlement des torts. Selon lui, le système judiciaire bourgeois constitue au contraire une partie de l'ordre bourgeois de classe, il travaille au service de cet ordre, il est donc à écarter en même temps que cet ordre ; les luttes populaires ne devraient donc pas, pour leur part, formuler ou poursuivre leurs demandes en reprenant des notions ou des instruments d'un ordre qu'il s'agit pour elles de renverser.

Trois mois plus tard, Foucault participe à un autre débat, cette fois nontélévisé, avec deux leaders alors clandestins du parti maoïste interdit, la Gauche prolétarienne, Benny Levi et André Glucksmann ; l'enjeu du débat étant cette fois la « justice populaire ». Le transcrit a été publié dans la revue Les Temps modernes en juin 1972, en indiquant que la discussion entre les participants avait commencé « lors du projet, en juin 1971, d'un Tribunal populaire pour juger la police».

Foucault exprime alors ses objections au modèle du tribunal populaire que la Gauche prolétarienne souhaitait tenir, en reprenant des arguments en cohérence avec ceux de sa discussion avec Chomsky. Foucault soutient ici, en citant l'exemple des massacres de Septembre pendant la Révolution française, que la formalisation de la justice populaire par le moyen des tribunaux révolutionnaires peut avoir comme effet une amplification considérable de l'échelle et de la violence des actes spontanés de violence populaire, qui frapperont des

3. Foucault M., Freedom and Knowledge, Elders F., Claris Elders L. (eds.), Special Productions BV, Amsterdam, 2014. 
ennemis ou des oppresseurs, mais en même temps, immanquablement, bien d'autres victimes. Certes, Foucault ne met pas ici en question la légitimité de la colère du peuple, au moins dans ce contexte de guerre révolutionnaire, mais si celle-ci est donc censée être juste, et n'est ni à redouter ni à brider, le danger, selon Foucault, consiste plutôt dans sa manipulation ou sa perversion par des éléments tiers prétendument neutres (dans ce cas, les chefs de sections parisiens ou les cadres révolutionnaires).

Dans les textes dont nous disposons actuellement, Foucault ne se réfère plus par la suite à cette discussion, sauf en une occasion. Dans un entretien tenu en 1981 à Louvain-la-Neuve à l'occasion de son cours Mal faire, dire vrai : fonctions de l'aveu en justice, ses interlocuteurs se réfèrent en effet à son débat avec les maoïstes et lui demandent son avis sur les tribunaux révolutionnaires qui se tiennent alors en Iran sous le régime de Khomeini.

Foucault y répond alors, en rappelant ce débat de 1971 :

« J'ai alors fait une critique de cette notion de tribunal populaire. Dans ces mouvements émotionnels intenses qui demandent une intervention forte de la part des gens, il n'y a pas besoin de justice, il y a besoin de vengeance : ces gens veulent se battre ; ce contre quoi ils en ont, ce sont leurs ennemis. Il y a un fond de guerre sociale qui est encore très présent lorsque tout spontanément, les gens veulent lyncher quelqu'un qui, parfois, n'a commis qu'un vol. Il est perçu comme l'ennemi social et on veut l'abattre comme tel.

Les gens qui veulent fonder un tribunal populaire sur quelque chose qui est en réalité une guerre se trompent doublement. Ou bien en effet ils ne font pas ce que veulent les gens et ils ne font pas la guerre, ou ils font ce que veulent les gens et ils ne font pas la justice. Je dirai les choses d'une manière encore plus crue. Vous savez parfaitement que si on fait des jurys tout à fait populaires, la peine de mort sera appliquée à tout le monde, même aux voleurs de canards. Donc, il y a ce fond de guerre sociale : celui qui vole fait la guerre; celui qui est volé se bat contre celui qui vole. Il ne faut pas oublier cela. Alors, il faut avoir le courage de dire que la justice est faite pour empêcher cela, et non pour traduire cela. Le tribunal populaire traduit cela. Khomeini, c'est exactement cela. Cette discussion a été de nouveau mal comprise. Les gens y ont aperçu une apologie de cette justice qui n'aurait même pas la forme d'un tribunal populaire, et qui serait l'égorgement. Non, non...»

Entre 1971-72 et 1981, il est clair, en suivant ces deux textes, que la pensée de Foucault sur la justice (et/ou sur la lutte de classe) a évolué, au moins dans certains aspects. Foucault a cessé de voir dans les luttes - plutôt que dans le 
droit - le seul principe des analyses généalogiques. Ceci est à remarquer pendant le cours de 1976, «Il faut défendre la société », sans que tout le monde ne s'en soit aperçu. Pour autant, Foucault ne devient pas durkheimien et ne souscrit pas à l'usage de la juridification comme mécanisme de résolution de tout affrontement ou de friction. Il conseille même plutôt de s'opposer à cette tendance qu'il voit dans le programme des socialistes avant 1981. Son avis négatif sur les tribunaux populaires reste constant mais, bien qu'il ne trouve pas l'occasion en 1981 de le dire, les mobiles du soupçon semblent s'être modifiés. Il n'est plus certain que l'œil du paysan voie toujours juste, ni que l'instance judiciaire soit en toute circonstances un pur truquage.

Or, on peut tracer un déplacement quelque peu analogue, à travers cette même période, entre les deux traitements d'un texte identique dans les cours tenus respectivement au Collège de France en 1971 et en 1980. Il s'agit du CEdipe Roi de Sophocle. On a assez récemment appris que Foucault, lorsqu'il s'embarque dans son «trip gréco-latin » dans ses cours au Collège de France en 1980 avec de nouvelles problématiques et un nouvel agenda de recherche, commence justement par la relecture de ce texte qu'il avait d'abord commenté en 1970-1971, lors de son premier cours au Collège de France, puis dans ses conférences brésiliennes.

Dans le cours de 1970-71 sur les origines de la politique, de l'économie monétaire et du droit public, on retrouve la référence à la Grèce archaïque, prise dans une matrice généalogique de la guerre civile, des nouvelles inégalités, et de la lutte des classes. Foucault se situe ici dans les pas de la philologie marxiste de Pierre Vernant et d'autres, en mettant à l'épreuve des savoirs historiques, comme l'a expliqué Daniel Defert, des thèses généalogiques de Nietzsche. Une nouvelle justice, axée sur la notion de mesure, pacifie certes la guerre sociale, mais elle entérine les inégalités. La lecture de l'CEdipe met l'emphase ici sur le discrédit et la déchéance du pouvoir-savoir malin et rusé du tyran $E$ dipe, marquant le début symbolique d'une longue inconscience dans notre culture politique du fonctionnement des pouvoirs-savoirs.

Neuf ans plus tard, dans le cours de 1980 (et à nouveau dans Mal faire, dire vrai), l'analyse est reprise, tandis que les accents sont modifiés, déplacés, et parfois inversés. Le commentaire sur l'CEdipe fait plutôt valoir l'apparition d'un postulat positif, donné ici également par Foucault comme un trait fondateur du droit public de la culture occidentale, à savoir que l'exercice légitime de souveraineté doit être lié (selon des modalités qui sont historiquement très variées) à une " manifestation de la vérité », en sorte que par exemple le direvrai d'un esclave puisse par un procédé juridique (en l'occurrence la menace de supplice) entraîner la chute d'un roi.

Ces deux déplacements d'analyse parallèles à travers les années 1970 dans les conceptions de Foucault concernant, respectivement, le statut de la justice 
et celui de la vérité, sont sans doute tous les deux à la fois partiels et complexes, mais paraissent composer ensemble une progression cohérente. Il est donc intéressant de poursuivre en voyant en quoi cette inflexion se traduit dans la discussion sur les enjeux actuels du droit.

En 1976, comme nous venons de le voir, Foucault écrit que l'analyse politique est à réinventer, afin de se déprendre en premier lieu du modèle « juridico-discursif » de l'exercice du pouvoir, modèle qui est calqué sur l'essor et la forme historique effective des monarchies occidentales, dotées d'une autorité centralisée et munies d'un monopole de la force militaire, monarchies dont par ailleurs le droit se trouve armé et imposé par un pouvoir de vie et de mort. Foucault écrit alors, dans une phrase célèbre, que le recours persistant à ce modèle dans notre pensée politique, alors que les relations de pouvoir se sont entre-temps profondément remaniées, fait que nous «n'avons pas encore coupé la tête du roi ».

Or, en 1981, par une décision dont Foucault a été considéré parmi les partisans, le nouveau gouvernement de gauche supprime la peine de mort en France. En saluant cet événement, Foucault remarque que le dénivellement entre gouvernement et gouvernés s'est trouvé, de par ce fait, " réduit d'une tête "; manière de dire que cette reforme pénale a un sens plus vaste que le droit pénal et s'inscrit dans de ce que Foucault appelle le « droit public », c'està-dire, pour lui, la relation entre gouvernants et gouvernés et la nature et les fondements de l'autorité gouvernementale. Éliminer l'archaïsme de la guillotine a donc une signification extrêmement importante en termes de philosophie politique et de pratique dans la mesure où, selon Foucault, cette mesure implique la possibilité, précisément, d'une mise en cause des rapports de pouvoir qu'il considérait en 1976 occultée par l'ancien paradigme du « juridicodiscursif ».

$\mathrm{La}$ « victoire du possible » en 1981, selon Foucault, relève ainsi de deux éléments conjugués : un déplacement et une ouverture réelle dans les termes de l'« analyse politique ", ainsi que des expériences et engagements de nouveaux acteurs qui, dans un kairos du possible, reconnaissent l'occasion et l'opportunité d'avancer des luttes entamées jusqu'alors en dehors de la scène politique. L'adoption de mesures, comme la suppression de la peine de mort, allant à l'encontre du sentiment de la majorité, mais commandant, dans cette échéance, le respect des gouvernés, fait dès lors partie de ce que Foucault appellera ici une « logique de gauche».

Il serait sans doute intéressant de tenter d'approfondir, de retrouver, reconstruire et redire plus clairement en quoi, selon Foucault, cette « logique de gauche» devait consister. Les éléments qui suivent n'en donnent que quelques prolégomènes. 
Si l'on revient un peu en arrière, on voit que Foucault a publié en 1979 le commentaire pointu d'une émission de télévision, apparemment filmée dans un restaurant parisien, où le Garde des Sceaux Alain Peyrefitte discutait de ses soucis judiciaires avec des invités du public ${ }^{4}$. Foucault commence par remarquer que le gouvernement (néo)libéral du jour, par de pareilles initiatives publicitaires, semble chercher à promouvoir auprès du public une conception du système judiciaire comme service public, le judiciaire étant alors censé fonctionner à distance de la responsabilité politique exécutive (comme Foucault l'indique, dans la discussion télévisée, il semble que le public ne paraisse pas trop croire, ni trop vouloir croire à cette soi-disant séparation); alors que l'État, pour sa part, garderait entre ses mains ce symbole visible et permanent de son autorité qu'est la peine de mort. Foucault estime que les invités du public paraissent contents des deux parties de cette offre (le service public judiciaire et la guillotine), mais lui-même s'interroge pour savoir si ces deux éléments sont vraiment cohérents. En effet, est-ce que les citoyens voudront recourir à un service public qui a, parmi ses fonctions, celle de leur couper la tête ?

Foucault indique ici le premier de ce qui me semble être ses deux arguments majeurs contre la peine de mort (l'autre étant l'inadmissibilité de l'amalgame de pouvoir/savoir juridique qui accouple le pouvoir mortel de punir avec l'expertise ubuesque du criminologue) : à savoir que la peine de mort, symbole exorbitant d'un sur-pouvoir prédémocratique et régalien, est maintenue dans toute sa rigueur uniquement pour des bénéfices liés à une politique démagogique.

C'est pourquoi, deux ans plus tard, avec l'abolition de la peine de mort et le cérémonial habilement orchestré par Mitterrand qui en fait l'accomplissement historique de l'œuvre démocratique inachevée de la Libération, Foucault évoque à propos de cette conjoncture particulière le triomphe d'une « logique de gauche ». Selon lui, la suppression de la peine de mort sera acceptable par l'opinion publique dans ce contexte d'exception et d'accomplissement historique, si elle est comprise comme une réforme quasi-constitutionnelle relevant d'un exorcisme démocratique des traces de Vichy restées dans la culture politique et sociale des IV et ve Républiques (permettant par ailleurs de rallier la France, par ceci, aux acquis de la modernité de ses pays partenaires et voisins). On voit alors comment la refonte très partielle du droit pénal peut valoir comme symbole d'une reconsidération de tout le droit public.

4. Foucault M., "Manières de justice ", Le Nouvel Observateur, 743, 5-11 février 1979. Repris in Dits et Écrits III, Paris, Gallimard, 1994, pp. 755-759. 
Pourtant, comme Foucault devait aussi sans doute le pressentir, de tels moments de possibilité et d'opportunité historiques sont rares. En l'absence d'occasion pour de semblables créations stato-dramaturgiques, l'état de normalité politique risque d'offrir peu d'occasions pour une telle logique de gauche et on pourrait même tendre, comme on l'a en effet vu plus tard en France et ailleurs, vers une logique plus en phase avec les traits d'une culture populaire de pénalité de style américain, où des régimes sévères de sécurité et pénalité reposent tout autant sur une volonté de la majorité où la peine de mort est bien, pour certains, un service public apprécié, plutôt qu'une démarche étatique ou autoritaire. On reviendra sur cette question en conclusion.

\section{Foucault et le droit en général : plaies et accusations}

Surveiller et Punir, le livre le plus polémique de Foucault, a été aussi comme par hasard ! - la cible la plus fréquente d'objections polémiques assez souvent provenant d'auteurs classés à gauche. Aussi allons-nous essayer de restituer un peu mieux le champ des positions antagonistes où s'aventure ce travail, en étudiant les blessures et les provocations que l'ouvrage de Foucault est censé avoir commises en ces diverses matières, avant de revenir sur la question de l'exercice du pouvoir et de sa relation au droit.

Dans Surveiller et Punir, et dans les discussions préalables à ce livre, Foucault avait amorcé une critique de l'analyse de classe de la gauche marxiste, qui selon lui avait échoué à cerner et à résister à la tactique de classe bourgeoise au XIXe siècle, passant par la scission entre la plèbe prolétarisée et la plèbe non-prolétarisée par le moyen des systèmes policier et carcéral et leur gestion du milieu criminel. Une gauche qui, par ailleurs, utilisait sans aucune réticence les techniques disciplinaires (et autres) de l'ordre bourgeois pour ses propres besoins de formation d'une force d'avant-garde révolutionnaire, pour la correction de ses erreurs et pour l'élimination de ses concurrents et de ses adversaires, avec les conséquences que l'on arrivait à connaître et reconnâ̂tre de mieux en mieux, pour ce qui en était de l'URSS et de la Chine, autour de ces années 1970.

Or, en même temps, le libre sujet de droit, citoyen de la modernité occidentale, démocratique et humaniste, se trouvait, dans ce livre de Foucault, rudement traité de "sujet docile » des pouvoirs-savoirs de normalisation, pouvoirs instrumentalisés et rationalisés par des sciences humaines dont la criminologie fonctionnait comme l'exemple même de la bêtise.

Parmi l'audience plutôt rétive de ces leçons, on pourrait distinguer d'une part les orphelins du socialisme alors « réellement existant », qui se mettaient à cette période à devenir des défenseurs des Lumières; et d'autre part (mais 
avec beaucoup de recoupements), les nouveaux prédicateurs et jurisconsultes d'un Grundordnung démocratique, solidement établi sur le fondement des éléments des sciences humaines. Il y eut à cette période une vague d'aggiornamento du marxisme « occidental » qui s'efforçait, à la suite d'Althusser ou de Gramsci (parfois avec l'ajout d'une sociologie du droit durkheimienne et de la doctrine du sujet et de sa loi selon Lacan), de produire un modèle sagement démocratisé du socialisme, habilité à se battre sur le champ des luttes idéologiques, en se présentant comme savant et fiable pour gérer les changements sociaux par les instruments de la légalité tout en assurant le maintien du lien social fondé sur la règle de droit. Durant un certain temps, on traita donc Foucault de gauchiste régressif qui, dans ses analyses, désapprenait le langage du droit, au moment même où des théoriciens néomarxistes de pointe s'étaient mis à le (ré)apprendre.

En même temps, Surveiller et Punir, manifeste s'il en fut des luttes antidisciplinaires, muni de récits de luttes populaires oubliées du XIXe siècle contre les prisons, et qui va inspirer à cette époque une vague de jeunes chercheurs de talent réunis dans la revue néofoucaldienne Révoltes logiques, s'est trouvé être lu par une bonne partie de la gauche anglo-américaine comme un pur hymne (pessimiste et/ou nihiliste) au pouvoir et à l'impensabilité d'y résister.

Pendant à peu près vingt-cinq ans, une orthodoxie quasi-obligatoire pour un universitaire américain gauchisant obligera alors à dire qu'il manque à Foucault une conception de la résistance, et qu'il échoue à répondre au grand problème de notre temps, à savoir celui de l'« agency ", néologisme américain de ces années intraduisible (je le crois fortement) en français et qui veut dire " capacité d'agir » (et plus particulièrement, capacité d'agir à gauche). Grosso modo, tout s'est passé comme si les orphelins de la révolution introuvable avaient alors choisi de voir (avec ressentiment) en Foucault le reflet et l'écriture de leur propre sentiment d'impuissance.

Les accusations contre Foucault continuent avec l'édition, parue à l'étranger mais pas en France, de la leçon de 1978 sur la gouvernementalité, leçon dans laquelle une distinction est faite entre la rationalité gouvernementale et les fondations de la souveraineté, puis dans les années 1990 avec une diffusion plus large d'une information en anglais sur les cours de 1978-79 relatifs à la gouvernementalité et ses commentaires critiques à propos de la pertinence des théories de l'État. La remarque de Foucault sur « la tête du roi » est reçue comme un défi dans les années 1980 par la grande majorité de ses critiques à gauche, qu'ils soient post-althussériens, post-trotskistes et/ou habermassiens.

On dépeint alors Foucault en nihiliste nietzschéen (Jungkonservative, au dire d'Habermas lui-même) ravalant toute théorie politique (y compris la théorie de la souveraineté politique et sa légitimation, la théorie du droit et des 
droits et la théorie de l'État) au rang de fiction édifiante, et considérant la loi, le droit et les institutions juridiques comme des réalités de second ordre par rapport aux formes réelles de l'exercice du pouvoir dans les sociétés modernes, tout ceci sans proposer d'alternative en termes de légitimation des luttes de résistance ou d'un ordre meilleur.

Dans les cercles universitaires anglophones préoccupés de la sociologie «critique » du droit et d'une éventuelle politique démocrate et socialiste du droit, deux auteurs ont lancé en 1994 ce qu'ils appelèrent « la thèse de l'expulsion » (expulsion thesis), selon laquelle Foucault aurait « expulsé » le droit de l'analyse historique et politique de la modernité ${ }^{5}$. Un peu comme Jean Baudrillard qui, avec une dextérité de polémiste, avait traité les remarques de Foucault sur le pouvoir comme constituant elles-mêmes un système de despotisme intellectuel, l'historien du Grand Renfermement serait celui qui aurait banni les juristes de sa république ${ }^{6}$. Résultat, sous cette pluie de fins de nonrecevoir et d'interdictions en règles, les contenus de Surveiller et Punir concernant précisément le droit et le système de justice criminelle et pénale vont finalement avoir tendance à être passés sous silence durant des années.

\section{Foucault et le droit en général : confusions, positivités}

Que dire de tous ces reproches ? Notons d'abord que dans l'évaluation des positions de Foucault concernant le droit, il faut distinguer entre méthode et matière. En refusant de concevoir l'exercice du pouvoir à partir du modèle de la loi et du droit, on n'élimine pas pour autant le juridique de l'histoire, de la matérialité des relations sociales, ni des pratiques et relations de pouvoir et de gouvernement. Mais, pendant un long moment, on a néanmoins reproché à Foucault de cantonner l'analyse du pouvoir aux micropouvoirs disciplinaires, de faire l'économie du fait étatique, d'être aveugle à la centralisation étatique des pouvoirs et aux enjeux de résistance au niveau stratégique.

Foucault a alors établi, en répondant à ce premier reproche, que l'histoire de la gouvernementalité fournissait aussi une analyse du fait étatique, établissant une continuité d'analyse entre les échelles micro et macro et ce, sans avoir à postuler une essence ou une puissance causale intrinsèque à l'État lui-même. En retour, on lui a objecté que l'histoire de la gouvernementalité excluait toujours la dimension de la souveraineté et des fondements de sa légitimité - en particulier les enjeux des révolutions et de l’ordre démocratique.

Pourtant, à bien regarder les choses, Foucault propose au contraire que l'histoire des gouvernementalités soit enchevêtrée avec l'histoire des souverainetés : dans le passé récent, par exemple, il indique dans Naissance de la biopo-

5. Hunt A., Wickham G., Foucault and Law: Towards a Sociology of Law as Governance, Londres, Pluto Press, 1994.

6. Baudrillard J., Oublier Foncault, Paris, Éditions Galilée, 1977. 
litique comment la gouvernementalité du marché a pu fonctionner en Allemagne comme un moteur de recréation d'une légitimité étatique à partir de la réduction de celle-ci à zéro en 1945.

D’une façon plus générale, il serait sans doute utile de répertorier les nombreux écrits où la recherche de Foucault nous propose, quoique de façon éparpillée, un certain nombre d'analyses où il est accordé au droit justement son plein statut de réalité historique et de matière à analyser en termes de positivité.

\section{Positivité historique et généalogique du droit}

Sur le plan historique, on trouve à plusieurs reprises dans les analyses de Foucault des épisodes où le droit fonctionne comme lieu ou source d'invention et de mutation des modalités de savoir/pouvoir ou de gouvernement. Dans le cours brésilien de 1973 sur «La vérité et les formes juridiques», la technique d'enquête, l'invention des pratiques de droit ecclésiastiques et civiles médiévales, servent comme foyer de tout un ensemble de savoirs empiriques en Occident. Tout en se moquant du prestige trop pérenne d'un modèle de pouvoir juridico-régalien, Foucault reconnaît parfaitement que la captation centrale des pouvoirs judiciaire, fiscal et guerrier est l'opération fondatrice des puissances étatiques occidentales. Pareillement, dans ses cours sur la gouvernementalité, l'action et le processus légal apparaissent comme des caractéristiques électives du libéralisme, comme l'art de la limitation du pouvoir gouvernemental. Pourtant Foucault estime ici plus efficace, au niveau des pratiques, la formule « anglaise » et « radicale », voire utilitaire, de limitation du gouvernement par la représentation des intérêts, que ses équivalents «français» et « révolutionnaires » qui, selon lui, limitent le despotisme par l'exigence de faire valoir des droits.

De même, dans le portrait fait par Foucault de la version allemande du néolibéralisme comme ordolibéralisme, un interventionnisme juridique indéfini fait partie de la caractéristique essentielle d'un style de gouvernement actif, consacré à sauvegarder un espace de jeu non-naturel de libre concurrence. Ainsi, en voulant faire valoir le caractère spécifique de la gouvernementalité comme type d'objet historique, Foucault ne renie pas le contrepoint historique continu entre le registre des arts de gouvernement (avec leur économie, leur efficacité, leur principe de rationalité acceptable) et celui du problème récurrent des fondements légitimes de la souveraineté, ce second terme faisant référence, comme le fait remarquer Foucault dans sa première leçon du cours de 1980, leçon qui précède justement la lecture d'CEdipe que nous avons citée ci-dessus, à des procédés qui ont des fonctions non-utilitaires de manifestation de vérité. 


\section{Le droit public}

Il est aussi significatif que, à partir de 1976, une thématique du «droit public » apparaît d'une manière non-systématisée, mais significative, dans les différents cours au Collège de France. Le thème est souvent abordé en lien avec des évocations au théâtre (d'Athènes, de Shakespeare, de Racine et Corneille, de l'Allemagne baroque) comme scènes de leçons sur le droit public, ou bien sur les coups d'État, dénominations sans doute plus ou moins alternatives du même enjeu. Il est enfin utile de remarquer que Foucault, malgré quelques réticences (évoquées ci-dessus) n’a pas eu tendance en général à renier en théorie ou en pratique la pertinence des droits. En 1971, moment de la discussion avec Chomsky, il suffit pour s'en convaincre de consulter les demandes faites par le GIP au sujet des droits (d'ailleurs très élémentaires) des prisonniers de droit commun. Pierre Vidal-Naquet, invité par Foucault à participer au lancement du GIP, a été un officier et un militant de la Ligue des Droits de l'Homme. À propos de l'affaire de l'extradition en Allemagne de l'avocat Klaus Croissant, Foucault remarque que l'on n'est pas tenu de croire naïvement à la générosité des États qui ont décerné autrefois et par raison d'État quelques protections à des exilés politiques pour se résoudre à demander aujourd'hui de la part de ces États le respect strict de ces mêmes garanties. Il n'y a pas à négliger l'usage stratégique d'un recours juridique, du fait de sa généalogie douteuse.

Dans « Le citron et le lait », texte d'hommage à Philippe Boucher, journaliste spécialisé dans les affaires judiciaires, Foucault insiste sur la non-réductibilité mutuelle des principes de l'ordre et de la loi, alors que, et même précisément parce que, dans les pratiques de pouvoir il y a justement fortement tendance à les amalgamer ${ }^{7}$. À diverses reprises, Foucault évoque le surgissement des demandes de nouvelles formes de droit et lui-même propose comme objet de travail collectif l'élaboration de nouvelles conceptions du droit, comme des droits anti-disciplinaires ou bien des droits des gouvernés.

\section{Les séquelles de Surveiller et Punir : les mutations du droit et de l'histoire de la gouvernementalité}

En ce qui concerne spécifiquement le droit pénal, il faut se rappeler que la recherche dans le domaine pénal de Foucault et de certains des chercheurs qui étaient autour de lui ne s'arrête pas avec la parution de Surveiller et Punir. Les traces de ce travail (collectif) de recherche se trouvent pour la plupart hors des livres et des cours que Foucault donne publiquement en France, mais il n'en existe pas moins. Le texte capital, prolongeant des indications déjà données à la fin du cours de 1976 au sujet des écoles de criminologie belges et italiennes de « défense sociale », est sans doute le cours lu et publié au Canada en 1978,

7. Foucault M., «Le citron et le lait». Repris in Dits et Écrits III, Paris, Gallimard, 1994, p. 695.. 
«L'évolution de la notion d'“individu dangereux" dans la psychiatrie légale du XIX ${ }^{\mathrm{e}}$ siècle ». Foucault explique ici que le parasitage du droit pénal par des savoirs-expertises disciplinaires ou normatifs n'a pu avoir lieu qu'à partir d'une refonte fondamentale de l'ontologie du droit lui-même. Cette perspective a été poursuivie dans les travaux de ses collaborateurs comme François Ewald, Pasquale Pasquino, Giovanna Procacci, Catherine Mevel-Donzelot, Daniel Defert, Robert Castel et Jacques Donzelot.

Cette refonte du droit tient notamment à l'avènement d'une nouvelle conception du droit social et à l'invention d'une conception de la responsabilité sans faute, ayant des applications à la fois dans le droit civil (accidents du travail, assurances) et dans le droit criminel (réorientation de la justice pénale de la sanction des actes vers l'identification et le traitement préventif de l'individu dangereux). Cet avènement annonce une société à la fois libérale, assurantielle et sécuritaire, une société qui vit dangereusement en même temps qu'elle gère ses risques. Cet ordre, qui à la fois produit et consomme des libertés, s'est montré par la suite tout à fait capable de détendre les disciplines, d'assouplir ou de démultiplier les normes, mais en même temps cet ordre a rendu possible une dérive, un engrenage sécuritaire et démagogique.

Ces recherches sur les mutations du droit social, du droit du travail et $\mathrm{du}$ droit des assurances faisant partie intégrante d'une histoire de la gouvernementalité des États-Providence, font ainsi apparaître un réseau de savoirs, de techniques et de problématiques (solidarité, risque, sécurité) qui sont tout à fait pertinentes pour une histoire du présent en 1979, mais aussi et encore pour le nôtre en 2014, car il est peu probable que l'on puisse faire le diagnostic des formes du néolibéralisme d'aujourd'hui sans être capable de faire la généalogie des États de providence d'hier et (pour une partie encore non négligeable) d'aujourd'hui. Ces analyses, qui ont été dispersées depuis 1984 (sinon avant) du fait de la diaspora des individus et des aléas de carrière, seraient certainement utiles pour une histoire du présent si elles étaient rassemblées et analysées.

\section{Foucault en 1980-84 : droit et pénalité dans le «trip gréco-latin »}

Les cours de Foucault de 1978-79 sur la gouvernementalité dépeignent les capacités d'innovation des néolibéralismes de son temps, indiquant la nécessité pour le socialisme de s'inventer sa propre rationalité de gouvernement, et on regardera ci-dessous si ses analyses peuvent servir à étudier la suite de ces histoires. Auparavant, il faut rappeler que Foucault lui-même n'a pas poursuivi après 1979 ses analyses des gouvernementalités modernes et actuelles, et s'est mis à faire un peu autre chose. Pourquoi ? Quelle est donc l'idée qui préside à l'organisation du cycle de cours de 1980 à 1984 (Louvain 1981 et Berkeley 1983 inclus), un ensemble de recherches dont on voit bien maintenant que les enjeux dépassent largement la portée des deux volumes parus en 
1984 : un cycle dont Foucault indique en 1984 qu'il touche à sa fin, sans avoir le temps ni de le conclure ni de donner une vue d'ensemble de ses objectifs et de ses résultats. Ces recherches sont donc inachevées et pour une large partie n’ont été disponibles que très récemment. Elles n'ont guère (ou peu) fait un apport direct aux enjeux juridico-pénaux de leur époque. Pouvons-nous néanmoins y trouver un apport pour nos enjeux de maintenant ? On va tenter ici rapidement de démontrer que oui.

Pour identifier le cœur thématique de ces recherches des années 1980, on peut utilement commencer avec le texte de Dumézil que Foucault met en exergue dans sa seconde leçon de Louvain en 1981 : « aussi haut que l'on remonte dans le comportement de notre espèce, la parole vraie est une force à laquelle peu de forces peuvent résister ». À travers ces cours, c'est l'enquête sur les transactions et tractations avec la vérité (jeux, actes, régimes et pratiques de vérité, avec, dans chaque cas, les fonctions et les rôles correspondants du locuteur du dire-vrai) qui paraît être le fil rouge. Quatre notions-clés, imbriquées, développent alors cette thématique : alêthurgie (manifestation de la vérité, y compris notamment la notion d'une vie véritable ou véridique), véridiction (modalités du dire-vrai), aveu (énonciation hasardeuse de vérité sur soi), parrêsia (franc-parler ou courage de la vérité, pour les bénéfices de l'autre ou de la cité). La période dite «dernière » du travail de Foucault est souvent commentée en termes de ses soucis éthiques : elle pourrait aussi bien être surnommée sa période "aléthique », la période des problématiques de vérité.

Dès 1979, Foucault considère que l'axe des rapports entre « véridiction » et «juridiction » se trouve au cœur de sa problématique; et par juridiction, on peut considérer qu'il entend aussi bien le politique (ou le dire politique) que le droit (ou le dire juridique). Entre 1980 et 1984, on le voit s'intéresser également à la fonction du dire-vrai dans l'espace public culturel contemporain comme le montre son entretien avec Edmond Maire.

De même, il s'occupe de la lutte entre franc-parler et démagogie dans la démocratie comme le signale son affrontement avec les socialistes à propos de la Pologne, mais aussi la discussion avec R. Bono sur les apories et les choix qu'il faudrait « avoir le courage » d'étudier concernant la sécurité sociale et les coûts de la médecine ${ }^{8}$.

Dans le cadre de ces analyses, le rapport à soi et le gouvernement de soi, avec ses pratiques et techniques, apparaît comme la matrice de liberté et le foyer de résistance à la gouvernementalité 9 . Dans le premier cours de 1983, dont une partie a été éditée à part dans un texte célèbre, Foucault commente la

8. Foucault M., « "Un système fini face à une demande infinie” (entretien avec R. Bono) », in Sécurité sociale : l'enjeu, Paris, Syros, 1983, pp. 39-63. Repris in Dits et Écrits IV, Paris, Gallimard, 1994, pp. 367-383. 
conception de la question des Lumières chez Kant comme une problématisation parrhésiastique de l'actualité, et s'inscrit lui-même dans cette conception et filiation de la tradition kantienne.

Rappelons que la question d'une politique de la vérité surgit dans la réflexion de Foucault bien avant 1980, et qu'elle est apparue bien souvent en rapport avec des affaires judiciaires. Il en est ainsi par exemple du texte de 1976, «Une mort inacceptable », où il nous interpelle : " et si la tâche politique de maintenant n'était pas de produire de la vérité, de l'objecter partout où c'est possible, d'en faire un point de résistance irréductible ? 10 »

Fabienne Brion et Bernard Harcourt ont fait justement valoir que Mal faire, dire vrai - le cours sur les formes de véridiction et de juridiction prononcé par Foucault à Louvain en 1980-81 - s’inscrit lui-même dans le droit fil de ses interventions et de ses engagements antérieurs, en particulier au sein du GIP, le Groupe d'information sur les prisons. C'est dans ce cours de Louvain en 1981, que les enjeux de la pénalité se trouvent insérés dans ce cadre des thèmes « aléthiques ».

En effet, dans Mal faire, dire vrai, à la fin d'une histoire traversant trois millénaires de l'aveu dans ses diverses manifestations, judiciaires et/ou pénitentiaires, apparaît, au temps des Lumières (donc à peu près en contemporanéité avec le personnage du révolutionnaire 11 et de l'homme double des sciences humaines qu'il a évoqué dans Les mots et les choses), la nécessité, pour une justice éclairée, d'un criminel qui avoue et qui participe comme sujet au processus de son jugement et de sa peine. Mais ce bon sujet criminel a tendance de nos jours à faire de plus en plus défaut, ce qui, dans l'optique de Foucault, risque d'entraîner une crise de la raison de punir.

À propos de ceci, on peut se rappeler ce que Foucault avait proposé dans un exposé devant des philosophes français en 1978, où il définit la critique comme une volonté de n'être pas gouverné (ou de ne pas l'être ainsi, ou autant); et que dans une intervention au Canada, à la même époque, il avait évoqué à l'occasion d'une conférence sur les droits des prisonniers l'idée d'une «volonté de ne pas être puni ».

On aurait aimé lui demander le rapport qu'il voyait entre ces deux volontés, tant la seconde semble de nos jours avoir fait moins d'avancées que la première. L' «âme moderne », le sujet de gouvernement formé à subir et accepter

9. Foucault M., Cours du 17 février 1982, in L'Herménentique du sujet : Cours an Collège de France (1981-1982), p. 241, 2001.

10. Foucault M., "Une mort inacceptable », préface à Cuau B., L'Affaire Mirval ou Comment le récit abolit le crime, Paris, Les Presses d'aujourd'hui, 1976, pp. VII-XI. Repris in Dits et Écrits III, Paris, Gallimard, 1994, pp. 7-9.

11. Foucault M., Cours du 7 mars 1984, in Le courage de la vérité. Le gouvernement de soi et des autres II. Cours an Collège de France (1984), 2008, pp. 169-172. 
les formes de pouvoir-savoir caractéristiques des sociétés de normalisation dont parle ironiquement, et parfois un peu cruellement, Surveiller et punir, semble bien correspondre au sujet capable d'admettre et de subir les punitions pénales qui relèvent de cet ordre. Foucault semble nous indiquer que ce visage tracé dans le sable du temps serait en train de s'effacer.

Sur la base des textes actuellement disponibles, il ne semble donc pas qu'il $\mathrm{y}$ ait une philosophie foucaldienne du droit (bien que des élèves de Foucault aient tout à fait le droit d'en construire une, selon leurs idées). Suggérons toutefois que le lien entre véridiction et juridiction est un enjeu majeur et profond dans la pensée de Foucault. Le droit se trouve de fait impliqué de façon récurrente dans toutes les histoires que raconte Foucault sur les jeux indéfinis de vérité où se trouvent impliqués des puissances, des savoirs et des libertés.

\section{Néolibéralisme et pénalité : la prescience de Foucault ?}

Parmi les moyens de penser les déplacements des enjeux et des conjonctures entre 1975 et 2014, nous disposons des éditions posthumes des cours de Foucault qui ne cessent de retenir l'attention de nouveaux lecteurs. La parution en 2005 des cours de Foucault sur le libéralisme et le néolibéralisme lui a d'ailleurs valu à titre posthume des hommages quant à sa capacité de prescience et de prémonition. Mais, peut-être Foucault était-il tout simplement plus attentif, un peu plus averti et un peu moins en retard que ses contemporains vis-à-vis de son actualité : et les plus attardés en cette matière, n'ayant pas pris le temps de s'attarder sur l'histoire, ont maintenant tendance à croire que le néolibéralisme serait une invention des années 1975-80.

S'agissant spécifiquement des évolutions pénales des deux décennies depuis la mort de Foucault, on peut se demander ce qui peut être compris à la lumière de ses idées, ou alors relever des raisons pourquoi il faut aller chercher ailleurs, ou bien encore se mettre soi-même en quête d'une explication.

On a pu remarquer que l'ascendant gouvernemental du néolibéralisme aux États-Unis n'a aucunement amoindri la punitivité de cette société : on y a bien vu le contraire. Paradoxe, contradiction, ou logique interne des doctrines du libre marché ? Il est frappant qu'en 1979, Foucault, dans son survol des idées néolibérales de l'école de Chicago, se soit attardé précisément à étudier les conceptions économiques de Gary Becker sur les crimes et les punitions. Il fait remarquer que selon Becker, les sociétés ont intérêt à tolérer un certain nombre d'illégalismes : un certain nombre, c'est-à-dire un nombre supérieur à zéro. La tolérance zéro, avec sa surenchère récente de rigueur préventive, ne serait donc pas une idée néolibérale.

Faut-il alors supputer, pour retrouver la logique de ces pratiques, d'autres principes de gouvernementalité libérale que Foucault aurait omis en 1979 de 
dégager ? Par exemple, une dépendance originaire, depuis les débuts de la physiocratie, entre la naturalité, la liberté économique et un activisme exacerbé du droit pénal en faveur de la propriété ? Il est difficile de voir les preuves d'une telle nécessité, en tout cas elles ne paraissent évidentes ni dans le texte de Foucault, ni (à ma connaissance) ailleurs. Si on essaie par exemple de comprendre le rétablissement de la peine de mort aux États-Unis depuis la fin des années 1970 - pour autant que je sache, non discuté par Foucault avant sa mort -, on peut trouver que le livre récent de David Garland (autrefois lecteur attentif de Foucault) en propose une analyse pertinente, bien que dans les apparences éloignée de Foucault, en termes non pas d'une logique d'économie libérale, mais d'une culture politique démocratique et républicaine fortement décentralisée (un fort soupçon anti-étatique ayant été évoqué, en fait, par Foucault comme trait pérenne du « libéralisme » politique américain), où des mécanismes démagogiques locaux sont susceptibles de mobiliser un appui populaire pour une justice sévère sur des bases socio-historiques en partie esclavagistes ${ }^{12}$.

D'autres lecteurs ont, suivant la ligne d'analyse de Robert Castel ${ }^{13}$ (qui, comme Jacques Donzelot, est un bon connaisseur des innovations gouvernementales aux États-Unis), repris à Foucault ses analyses des «dispositifs de sécurité » comme outillages de la gouvernementalité occidentale depuis l'ère de l'État de police, y trouvant des moyens pertinents pour rendre intelligible une gamme de pratiques préventives orientées vers des populations-cibles censées être soit « à risque », soit porteuses de risques.

Les études de Foucault sur le néolibéralisme de son temps (et avant) restent donc une importante ressource pour la réflexion sur nos temps présents. Elles ne sont pas pour autant une clef suffisante pour tout comprendre. Malgré les tendances actuelles qu'on trouve chez certains auteurs, le néolibéralisme n'est pas un principe d'explication universel, pas plus que la biopolitique, ou le sécuritaire. Rappelons d'ailleurs que dans les analyses de Foucault il n'y a pas une seule, mais plusieurs variantes de néolibéralisme - allemand, français, américain. Et que la globalisation (mot, qui paraît-il, n'était même pas encore passé dans la langue courante du vivant de Foucault) n'a pas encore aboli ces différences, pas plus que bien d'autres.

12. Garland D., Peculiar Institution: America's Death Penalty in an Age of Abolition, Harvard University Press, 2010.

13. Castel R., La Gestion des risques. De l'anti-psychiatrie à l'après-psychanalyse, Paris, Éditions de Minuit, 1981 ; et Castel F., Castel R., Lovell A., La société psychiatrique avancée. Le modèle américain, Paris, Grasset, 1979. 


\section{Penser l'actualité, avec et sans Foucault : propositions}

Foucault lui-même a dit - en l'occurrence à propos de l'Iran - qu'il ne savait pas prédire le passé, ni faire l'histoire de l'avenir. Sa prescience n'allait pas, par exemple, jusqu'à prédire l'ascension de la tolérance zéro. Ses textes ne sont donc certainement pas un moyen de divination des temps futurs.

En revanche, la devise kantienne de sapere aude, «Aie le courage de te servir de ton propre entendement », doit nous inviter à penser nous-mêmes le nouveau, soit avec des moyens conceptuels à notre disposition qui conviennent à la tâche, soit s'il le faut, en s'en inventant d'autres. C'est pourquoi, au mieux, peut-être, pour qui le veut, essayer de penser notre actualité se fait à la fois avec et sans Foucault; sans manquer d'utiliser les outils qui servent entre nos mains, ne calquons pas pour autant l'intelligence d'un réel nouveau sur d'anciennes recettes. À ce propos, et pour terminer, voici quelques modestes propositions pour des lignes de recherche futures.

\section{Néolibéralismes et illégalismes}

La publication récente du cours de 1973 sur La société punitive attire notre attention sur une thématique majeure de Surveiller et Punir, assez largement oubliée (ce livre étant bien plus souvent dénoncé que lu) : les « illégalismes ». L’Ancien Régime offrait aux États et classes sociales des occasions diverses de détourner, de court-circuiter ou de truquer les lois à leur profit : contre ou en-dessous des lois, il y a eu les illégalismes divers et respectifs de la noblesse, de la bourgeoisie, de l'artisan et du menu peuple. Le lecteur marxiste, se plaignant du manque chez Foucault d'un principe ou d'une force de résistance au pouvoir étatique, semble avoir oublié ou fait l'impasse sur toutes ces résistances sournoises à l'ordre juridique, et leur réaménagement systématique, avec le plein avènement de la société de marché, au profit d'une classe : thème majeur du livre de Foucault, qu'on retrouve, exposé en détail déjà en 1973, dans La société punitive.

Il serait dès lors intéressant de relire dans cette optique, avec Foucault mais aussi en se souvenant des aléas récents du marché, les réflexions de Gary Becker sur l'économie des crimes et des châtiments. Admettons un moment avec Becker qu'il y a un taux minimal d'illégalismes (taux supérieur à zéro) audessus duquel la suppression des infractions ne serait plus rentable. Ce taux est-il le même pour des manipulations boursières, des infractions de stationnement de voitures, des graffiti ou des petits vols ? Si ce n'est pas le cas, quelles sont donc les différences entre ces taux?

Par ailleurs, comment actualiser, après le krach de 2008, le contraste très marqué que Foucault repérait entre les politiques de réglementation et d'enca- 
drement juridique de la concurrence prônées respectivement par les néolibéralismes allemand et américain (politique de réglementation active en faveur de la concurrence dans un premier temps, chez les Allemands; politique de déréglementation, à partir des années 1970-80, chez les Américains, suivis par les Britanniques) ? Pour mettre à jour l'analyse ponctuelle, sinon précoce, que Foucault fit alors du néolibéralisme, il serait sans doute pertinent de s'inspirer de ses analyses antérieures sur les illégalismes capitalistes et leur symbiose avec la règle du droit.

\section{Aveu et parrbésia des intellectuels spécifiques}

Nous avons vu que le « dernier » Foucault s'intéressait à la pratique de la parrhésia, du courage de la vérité, au présent autant qu'au passé. Cet intérêt et les obligations éventuelles qui pouvaient s'ensuivre pouvaient correspondre à des conduites de dénonciation qui ne sont pas forcément inédites, et pas forcément étrangères à une éthique préalablement existante de l'« intellectuel public ». Il y a eu le cas du docteur Édith Rose, la psychiatre de la prison de Toul, auteure d'une lettre publique au Président de la République, Georges Pompidou, où elle dénonçait une série de violences sur des détenus. Foucault joua alors un rôle de relais et d'amplificateur de cette critique personnalisée. Madame Rose paya de sa carrière, semble-t-il, le prix de son dire-vrai. Une décennie plus tard, Foucault remarque dans un cours sur les éthiques grécoromaines que le rapport de soi à soi gît au fond dans la capacité de résistance à la gouvernementalité ; il se peut qu'il y ait, dans cette réflexion, un hommage à ceux pour qui le rapport à soi passe par la problématisation de l'«intolérable » dans ce qu'on fait soi-même - un hommage qui souligne l'importance de ce type de résistance.

\section{Foucault et Badinter: jeu des conduites, cadre du droit?}

À l'encontre de quelques idées reçues, Foucault retire en 1983 de ses lectures de Platon le principe que les philosophes n'ont pas à donner des lois à leur cité. Sa lecture de la lettre de Platon sur ses voyages à Syracuse et sur les conditions de possibilité d'une parrbésia politique en diverses circonstances pouvait être alimentée par quelques expériences contemporaines et personnelles, et s'y référer tacitement; il est douteux, par exemple, que si Foucault évoque, dans le cours de 1983, l'œuvre de Jan Patocka - philosophe tchèque et militant de la Charte 77, décédé en 1978 après un interrogatoire policier -, ce soit uniquement en raison de sa réflexion érudite sur Platon.

En 2008, à l'occasion d'un colloque public sur Foucault, Robert Badinter, alors sénateur et président du conseil constitutionnel, a tenu un discours qui dénonçait avec la plus grande véhémence la législation sécuritaire et pénale du gouvernement français contemporain. Le philosophe et le juriste ont été des 
alliés, trente années auparavant, pour dénoncer publiquement, chacun avec ses propres raisonnements, la peine de mort. Foucault a salué l'acte de Badinter, qui supprima cette peine en 1981. Après 1981, il parait que Foucault (lui qui avait refusé deux fois, pour des raisons de " coupe-tête ", de déjeuner avec des présidents de la République) a ensuite consenti à agir quelques fois comme conseiller privé de Badinter, dans ses fonctions de ministre de justice. On sait que Foucault et Badinter travaillaient ensemble, au moment de la mort de Foucault, à un projet de création d'un centre de recherche en philosophie et droit. Badinter a écrit en 1986, dans le volume d'hommages à Foucault publié par la CFDT :

\begin{abstract}
«Au cours de nos dernières conversations, Michel Foucault soulignait la nécessité de mieux appréhender dans une société laïque et pluri-culturelle l'importance de la règle de droit et de sa fonction architecturale, transcendant le normatif pour devenir, de l'édifice social, l'arc-boutant : celui que des forces opposées soutiennent et qui assure l'équilibre de l'ensemble. »
\end{abstract}

Foucault pensait-il alors effectivement cela ? Peut-être. L'idée d'un régime de droit qui aurait pour vocation de protéger une société de différences, un pluralisme de conduites, peut paraître un peu en retrait par rapport à l'évocation, à la fin de Surveiller et punir d'un « grondement de la bataille » autour de la justice pénale. Aujourd'hui, cette même idée peut paraître un peu moins banale ou évidente et, dans cette mesure, encore digne de notre écoute, comme le sont les considérations qui ont pu la fonder aux yeux du philosophe. Précisons qu'il s'agissait ici des idées de Monsieur et non de Madame Badinter. Il était encore possible alors, paraît-il, d'imaginer une république à croyances plurielles.

\title{
Conclusion : le possible alors et maintenant
}

Essayons de reprendre nos questions de départ pour conclure brièvement.

\section{Douter}

Sur la question de : que faire avec la pénalité - que faire, sinon la prison, Foucault avouait ne pas avoir de recette, mais il prônait la persistance du doute, de la persévérance dans l'inquiétude sur le bien-fondé de ce que nous persistons à faire, faute de mieux ou faute d'idées sur le mieux, à condition que cette inquiétude ne cesse pas d'être le mobile d'un travail. Pour ce qui est du devenir du possible d'alors, en matière de pénalité, il semble que, en France, grâce à quelques doutes ou un peu de mauvaise conscience (où le travail et l'intervention de Foucault ont joué un peu comme nous l'avons évoqué), pour un temps au moins, il y ait eu des réticences devant l'option de l'acharnement carcéral, au moins en comparaison avec ce qui s'est passé ailleurs. La politique 
d'asile de l'État français a pu aussi, pendant un certain temps, donner l'air d'avoir compris les propos de Foucault dans sa lettre adressée aux leaders de la gauche. Mais ensuite, on a l'air d'être parti à la dérive, depuis que sont intervenues des forces nouvelles ou renforcées allant dans l'autre sens, pendant que les tendances préalables en faveur du doute faisaient plutôt semblant de s'endormir.

Pour ce qui est du possible maintenant : il faut nous demander, d'abord, si ce possible serait le possible d'un réel. Quelqu'un, qui n'était pas Foucault, a dit avant-hier que les droits de l'homme ne sont pas une politique. Foucault, pour sa part, avait admis que les droits humains étaient et sont un enjeu politique, pour peu qu'on les considère comme synonymes des droits des gouvernés. Quelque part dans notre actualité, on a tiré un peu d'espoir quand on a vu une menace de la part de l'instance judiciaire américaine de fermer une prison en Californie, en raison des critères qui ont sanctionné le traitement des détenus à Abu Ghraib. Les droits de ces gouvernés qui se trouvaient être des prisonniers, seraient alors fondés, pour leur bien, sur les droits des victimes de torture soumises à une puissance militaire, dans une époque de guerres dites humanitaires. Une certaine idée du possible maintenant ne serait-elle pas alors la possibilité de ne pas avoir à espérer, de ne pas avoir à vouloir que cela ?

Le possible en 1981, dans l'idée de Foucault, était qu'avec un rehaussement du droit public, un redressement du statut des gouvernés, il s'ensuivrait un réaménagement et un élargissement des notions sur les modalités de réflexion et d'intervention des gouvernés dans le champ de la gouvernementalité. Si les circonstances se sont modifiées, la pertinence de cette idée n'est pas pour autant dépassée.

Dans un célèbre petit texte sur les boat people et la nécessité d'une solidarité des gouvernés, texte qu'on a pu lire comme la charte philosophique des ONG de notre temps, Foucault affirme pour son compte que les gouvernés ont le droit de s'occuper et d'intervenir dans des questions dites humanitaires qui auparavant étaient censées relever du monopole des gouvernements, à travers le droit international des États ${ }^{14}$. La solidarité des gouvernés corrigerait l'incurie et la paresse calculée des politiques et des gouvernements, du moins, ajouterait-on maintenant à la lumière des expériences ultérieures, tant que les ONG ne se trouvent pas impliquées, et éventuellement compromises, comme acteurs dans les jeux entre les pouvoirs. Y aurait-il une logique de symétrie (et cohérence ?) de cette notion de débordement activiste du côté des gouvernés avec le principe anti-démagogique par lequel Foucault saluait la suppression,

14. Foucault, on se rappelle, avait parmi ses rares prévisions anticipé pour nos jours une grande ère de migrations et d'exodes, dont les implications pour nos pratiques carcérales et sécuritaires ne cessent d'exiger une vigilance inquiète. Foucault M., « Face aux gouvernements, les droits de l'homme ", Libération, 967, 30 juin-1er juillet 1984, p. 22. Repris in Dits et Ecrits IV, Paris, Gallimard, 1994, pp. 707-708. 
vraisemblablement contraire à la volonté majoritaire, de la peine de mort ? Beaucoup de notre avenir politique pourrait dépendre du fait de savoir si cette logique peut valoir et se prolonger.

\section{Indocilité et inquiétude}

Être rétif vis-à-vis d'un gouvernement, se tenir « debout » et en «face ». Patience et impatience. Discuter, même conseiller, là où cela se peut, là où il $\mathrm{y}$ a capacité d'écouter. Objecter une vérité, demander des explications, là où il le faut : voilà deux modalités de parrhésia, dans l'espace privé et public, commentés dans les derniers cours de Foucault sur les cas de Socrate, Platon et de bien d'autres. Ne pas flatter le prince, ne pas flatter l'ami. Ne pas flatter démagogiquement le peuple, ne pas se satisfaire de ce jeu rhétorique qui est de convaincre l'autre de ce que l'autre pense déjà. Ceci est une éthique qui dépasse de loin le seul domaine d'application des enjeux juridiques, mais qui convient passablement à ce domaine. C'est ainsi que se termine un de ses textes de 1981 :

«Il est un danger que peut-être on n'évoquera pas : celui d'une société qui ne s'inquiéterait pas en permanence de son code et de ses lois, de ses institutions pénales et de ses pratiques punitives [...]. Il est bon, pour des raisons éthiques et politiques, que la puissance qui exerce le droit de punir s'inquiète toujours de cet étrange pouvoir et ne se sente jamais trop sûre d'elle-même. ${ }^{15}$ "

15. Foucault M., " "Punir est la chose la plus difficile qui soit" (entretien avec A. Spire)", Témoignage chrétien, 1942, 28 septembre 1981, p. 30. Repris in Dits et Écrits IV, Paris, Gallimard, 1994, pp. 208-210. 